\title{
Validez y fiabilidad del Sistema de Detección Precoz de los Trastornos del Desarrollo: 3 a 36 meses
}

\section{The validity and reliability of the System for Early Detection of Developmental Disorders: 3-36 months}

\section{Resumen}

En este artículo se presenta el Sistema de Detección Precoz de los Trastornos del Desarrollo (SDPTD), desarrollado en trabajos anteriores (Rico, 20I3; Alonso, 20I4). Este sistema se compone de siete cuestionarios, uno para cada corte de edad $(3,6$, 9, I 2, I 8, 24 y 36 meses). Se han diseñado estos cuestionarios para ser contestados por los padres. Para estudiar su validez, se ha aplicado el SDPTD a una muestra de 728 niños/ as (aproximadamente roo niños/ as en cada una de las siete secciones de edad). Como prueba criterio se ha utilizado la Escala de Desarrollo Merrill-Palmer-Revisada (MP-R). Un año más tarde, se ha verificado el estado de desarrollo de los/as niños/ as. Los resultados muestran un alto acuerdo entre padres y profesionales. La validez concurrente es alta aunque varía con cada corte de edad. En cuanto a la validez diagnóstica de un año, las tasas de sensibilidad y especificidad son lo suficientemente altas como para considerar el sistema como fiable, válido y adecuado para los fines previstos.

\section{Palabras clave}

Detección, trastornos del desarrollo, validez concurrente, validez diagnóstica.

\begin{abstract}
This article introduces the System for Early Detection of Developmental Disorders (referred to as SDPTD for its abbreviation in Spanish), a system developed in previous papers. The SDPTD is a developmental screening test that includes seven questionnaires, one for each cutoff of age $(3,6,9,12,18,24$ and 36 months). These questionnaires have been designed to be answered by parents. To study its validity, SDPTD was administered to a sample of 728 children (approximately Io० children in each of the seven cutoff age groups). A development scale known as Merrill-Palmer-Revised (MP-R) was used as a criterion test. The development state of the children was tested again one year later. The results show a high level of agreement between parents and professionals. The concurrent validity is high although it varies by cutoff age. Regarding the diagnostic validity a year after the original evaluation, levels of sensitivity and specificity are high enough to consider the system reliable, valid and suitable for screening purposes.
\end{abstract}

Screening, developmental disorders, concurrent validity, diagnostic validity.

\section{Keywords}

\section{Francisco Alcantud Marín <francisco.alcantud@uv.es>}

Centro Universitario de Diagnóstico y Atención Temprana de la Universitat de Valencia

\section{Yurena Alonso Esteban <yurena.alonso@uv.es>}

Departamento de Psicología Evolutiva y de la Educación de la Universitat de Valencia

\section{Darío Rico Bañón \\ <dario.rico@uv.es>}

Centro Universitario de Diagnóstico y Atención Temprana de la Universitat de Valencia

Para citar:

Alcantud, F. et al. (20I 5): "Validez y fiabilidad del Sistema de Detección Precoz de los Trastornos del Desarrollo: 3 a 36 meses", Revista Española de Discapacidad, 3 (I): IO7-I2I.

Doi: <http://dx.doi.org/IO. 55 69/23405 I04.03.01.06>

Fecha de recepción: I I-I I-20I4 Fecha de aceptación: 25-05-20I 5 


\section{Introducción}

Se entiende como trastornos del desarrollo una disfunción permanente o transitoria (neurológica, psicológica o sensorial) que hace que se desvíe el desarrollo del niño/a de forma significativa del curso normativo. Generalmente se detecta cuando el niño/a no alcanza hitos significativos del mismo. En la literatura científica se utiliza tanto el término genérico "trastornos del desarrollo" como "retraso en el desarrollo" ${ }^{2}$ como termino transitorio en edades tempranas hasta avanzar o establecer un diagnóstico definitivo. Los trastornos del desarrollo se definen como "una tipología variada de problemas que tienen su origen en la infancia, la niñez o la adolescencia y que pueden perdurar a lo largo de toda la vida”. (García, I999, p.28).

Estos trastornos también fueron definidos como "crónicos, severos y atribuibles al retraso mental, parálisis cerebral, epilepsia o autismo, porque tales condiciones consisten en alteraciones similares del funcionamiento intelectual general y de la conducta adaptativa y requieren servicios y tratamientos similares a los requeridos por tales personas". (Schopler, I983, p. I20). Hoy en día podría referirse a ellos como trastornos del Neurodesarrollo. Es decir, aquellas alteraciones en el desarrollo de funciones vinculadas a la maduración del sistema nervioso central que se inician en la infancia y siguen un curso evolutivo estable (Artigas-Pallarés, 20I I). Sin embargo, cuando solo se tienen evidencias de un retraso en el desarrollo o un trastorno del desarrollo sin conocer las causas y por la edad, aun sin tener un diagnóstico específico, se suele mantener el término genérico de "trastornos del desarrollo".

Con independencia del diagnóstico, las evidencias de las mejoras producidas mediante los programas de Atención Temprana cuando se interviene antes de los tres años son abrumadoras

I. Del inglés "Developmental Disabilities". 2. Del Inglés "Developmental Delay".
(Barnett, I995; Reynolds, Temple, Robertson, \& Mann, 200I; McCormick, Brooks-Gum, Buka, Goldman, \& Yu, 2006; Casto \& Mastropieri, ı986; Shonkoff \& Hauser-Cram, I987; Guralnick \& Bennett, I987; Guralnick, I989,I997; Guralnick, I998). Sin embargo, hoy en día aún son muy numerosos los/as niños/ as que llegan a la edad escolar presentando un trastorno del desarrollo sin diagnóstico previo $y$, consecuentemente, sin haberse beneficiado de los servicios y programas de Atención Temprana. Así, por ejemplo, en USA, se constata que antes de entrar en la escuela infantil se identifican menos del $30 \%$ de los/as niños/as con trastornos del desarrollo (Glascoe \& Macías, 2003; Palfrey, Singer, Walker, \& Butler, I987). Las razones que se argumentan son diversas, por una parte unos apuntan hacia la carga asistencial de los servicios de pediatría en atención primaria. Consecuencia de la cual resulta difícil, por no decir imposible, desarrollar una exploración completa del desarrollo de todos los/as niños/ as atendidos. Otros apuntan hacia la falta de formación del pediatra que le llevaría a no utilizar sistemas normalizados (Smith, I978; Hamilton, 2006). La mayoría de ellos utilizan, como mucho, el juicio clínico, aunque se sabe que con este método no se detectan más del 30\% de niños/as con trastornos del desarrollo y menos del 50\% si se trata de problemas conductuales y emocionales (Alvarez \& Giner, 2007). Todo parece indicar, en definitiva, que solo se atiende sobre el $2 \%$ de niños/as menores de 3 años, frente al $5 \%$ de niños/as que se detectan con necesidades similares en preescolar (Sices, Feudtner, McLaughlin, Drotar \& Williams, 2003).

Este estado de conocimiento ha llevado a que las organizaciones profesionales y las entidades públicas responsables de la salud infantil, hayan desarrollado programas específicos de control del desarrollo en el/la niño/a, en los que se especifica la conveniencia de utilizar sistemas de screening o detección precoz de manera sistemática en diferentes momentos clave del desarrollo del niño/a. El problema parece radicar en la falta de accesibilidad a los instrumentos de detección y la falta de recursos para su administración. En nuestro país existen algunas experiencias en el caso de trastornos específicos, 
como es el caso del programa de detección de los Trastornos del Espectro Autista, desarrollado en Castilla-León (Canal, et al., 2006). Otro sistema desarrollado en nuestro país de uso extendido entre los especialistas en pediatría es la tabla Haizea-Llevant. Esta tabla derivada del Test de Denver consiste en un listado organizado de ítems representativos de los hitos evolutivos más significativos en el desarrollo del niño. Es el resultado de dos estudios o programas, el Estudio Llevant y el Programa Haizea, cuyos datos de referencia se obtuvieron de una muestra representativa de I.702 niños/as de Cataluña y 8I7 niños/as del País Vasco respectivamente. Esta tabla muestra el desarrollo cognitivo, social y motor de niños/as de o a 5 años (Fernández, I 988 y Fernández, E., Fernández, J., Fuentes, Rueda, I99I).

En los últimos años están tomando mucha fuerza el desarrollo de los sistemas de screening basados principalmente en las respuestas de los principales cuidadores del niño/a. En la mayoría de las investigaciones sobre la validez y fiabilidad de estos sistemas se informa de un acuerdo consistente entre las evaluaciones de profesionales y de padres/madres, en particular cuando estos últimos respondían a cuestiones sobre el desarrollo actual (Squires, Potter \& Bricker, I998). La información dada por los padres sobre el desarrollo de sus hijos/as ha sido utilizada en muchas de las escalas de desarrollo "clásicas" junto con la evaluación profesional (Inventario de Desarrollo Battelle -BDI-, Inventarios de Desarrollo Comunicativo MacArthur, Escala de desarrollo psicomotor de Brunet-Lézine, Escalas de desarrollo MerrillPalmer, Escala adaptativa Vineland, etc.). En este sentido, ya existen cuestionarios de cribado o detección precoz validados en Estados Unidos y España basados exclusivamente en la información recibida por la familia y con excelentes propiedades psicométricas (Parents' Evaluation of Developmental Status -PEDS-, The Modified Checklist for Autism in Toddlers -M-chat-, Ages \& Stages Questionnaires -ASQ-, y otros...).

Se entiende que los instrumentos de cribado están diseñados para identificar o detectar a niños/as que puedan necesitar una evaluación más exhaustiva. Suelen ser test o listas de chequeo breves (Aylward, I995; Hamilton, 2006; Melchers et al., 2003). La ventaja más obvia de este tipo de instrumentos es el coste reducido frente a la administración de este mismo tipo de pruebas por profesionales. Otra ventaja práctica que se les atribuye a este tipo de cuestionarios es que, según el estudio de Hickson et al., los padres/madres están más preocupados por el comportamiento y desarrollo de su hijo/a que por las cuestiones médicas y sin embargo no suelen consultar con su pediatra este tipo de preocupaciones (Hickson, Alteimer, \& O'connor, I983). De este modo cuando los/ as padres/madres contestan los cuestionarios de detección se consiguen efectos positivos adicionales, como por ejemplo, una mayor conciencia de la importancia del desarrollo evolutivo de sus hijos/as conociendo los hitos más importantes de su desarrollo.

Los sistemas de cribado del desarrollo son una medida preventiva (Hix-Small, Marks, Squires, \& Nickel, 2006) y en consecuencia son también competencia de los programas de Atención Temprana. Detectando de forma precoz permitimos también una intervención precoz que redundará en mayores beneficios para la familia y el niño/a a largo plazo y consecuentemente se rentabilizará la inversión económica necesaria para su implantación, desarrollo y mantenimiento de los programas de Atención Temprana (Rydz et al., 2006).

El Sistema de Detección Precoz de los Trastornos del Desarrollo (SDPTD) aquí presentado se basa en tres etapas o niveles. El primer nivel consiste en contestar un cuestionario adaptado a la edad del/la niño/a. Este cuestionario es auto-administrado por los propios padres/ madres. Estos cuestionarios pueden encontrarse en formato impreso o pueden administrarse por medio de un sistema web. El SDPTD se ha diseñado para ser administrado por medio de una aplicación web de forma que el coste de la administración por unidad es mínimo. Además, el sistema ofrece como beneficio añadido el uso de videos demostrativos de las conductas evaluadas con lo que la accesibilidad a los mismos se incrementa de forma significativa 


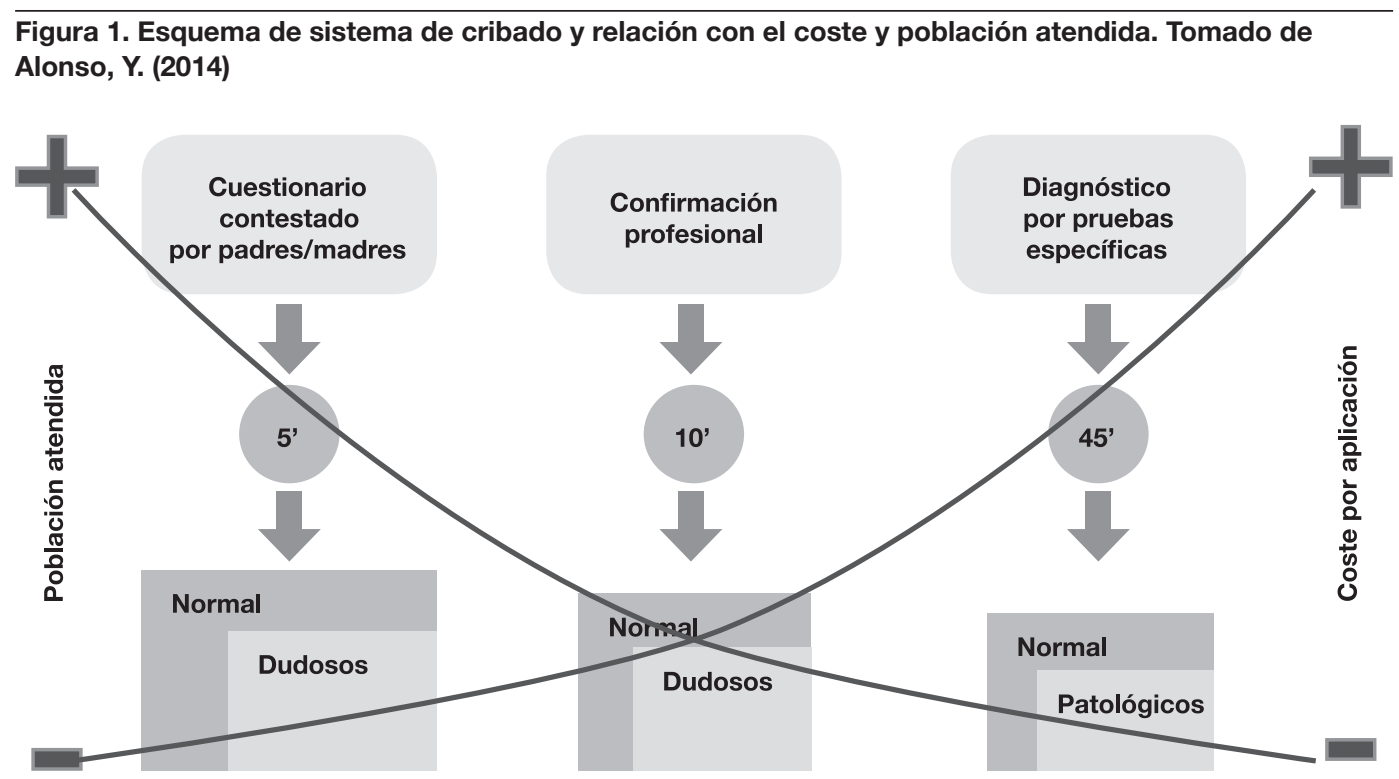

llegando al máximo de población. El segundo nivel del sistema es la confirmación profesional, que debería realizarse solo sobre los casos positivos del paso anterior y en la misma consulta del pediatra. Por último, el tercer paso sería la derivación a un centro especializado y solo recaería sobre una población mínima que hubiera sido detectada en los dos pasos anteriores. $\mathrm{La}$ imagen de la figura I, intenta evidenciar la relación inversa que debe haber entre el coste de la aplicación por familia evaluada y el número de familias que se deben evaluar.

El objetivo de este artículo es dar a conocer los resultados globales de la aplicación piloto realizada tras el proceso de construcción del SDPTD.

\section{Método}

\subsection{Instrumentos y cuestionarios}

La administración de un cuestionario de estas características debe ser obligatoriamente breve.
El objetivo del SDPTD es cubrir un periodo de edad en el que aún no existen instrumentos de detección de trastornos específicos. Del análisis de la literatura existente se ha detectado la falta de consenso en los cortes de edad más relevantes, así como sobre las áreas del desarrollo a describir. Así, encontramos en la literatura autores que diferencian el primer mes, como periodo neonatal, del resto del primer año de vida que dividen en tres periodos; los cuatro, los ocho y los doce meses. (Gesell, I974; Osofsky, I987; Horowitz, I989; Illingworth, I992). Por el contrario, otros autores presentan la información por meses o trimestres naturales (Aguirre, 200I) y por último, en el ámbito pediátrico, también existen autores que han hecho el esfuerzo de ajustar la exposición de hitos a las visitas de los programas de vigilancia del desarrollo del/la niño/a sano (Álvarez, Soria, \& Galbe, 2009; Olhweiler, Silva, \& Rotta, 2002).

Por otra parte, es importante caer en la cuenta que muchas conductas de las que se proponen observar son multidimensionales y en su desarrollo se ve implicada más de un área. En este sentido, solo se ha considerado el área 
Tabla 1. Número de ítems por cuestionario y área del desarrollo evaluada

\begin{tabular}{|l|c|c|c|c|c|c|c|}
\hline Áreas & $\begin{array}{c}\mathbf{3} \\
\text { meses }\end{array}$ & $\begin{array}{c}\mathbf{6} \\
\text { meses }\end{array}$ & $\begin{array}{c}\mathbf{9} \\
\text { meses }\end{array}$ & $\begin{array}{c}\mathbf{1 2} \\
\text { meses }\end{array}$ & $\begin{array}{c}\mathbf{1 8} \\
\text { meses }\end{array}$ & $\begin{array}{c}\mathbf{2 4} \\
\text { meses }\end{array}$ & $\begin{array}{c}36 \\
\text { meses }\end{array}$ \\
\hline Motriz & 6 & 4 & 6 & 4 & 4 & 2 & 4 \\
\hline $\begin{array}{l}\text { Audición } \\
\text { Lomunicación } \\
\text { Lenguaje }\end{array}$ & 3 & 3 & 2 & 5 & 2 & 3 & 4 \\
\hline Socio-Emocional & 1 & 3 & 2 & 4 & 3 & 4 & 3 \\
\hline Cognitiva & 2 & 1 & 4 & 3 & 2 & 2 & 1 \\
\hline Total & $\mathbf{1 2}$ & $\mathbf{1 1}$ & $\mathbf{1 4}$ & $\mathbf{1 6}$ & $\mathbf{1 1}$ & $\mathbf{1 1}$ & $\mathbf{1 2}$ \\
\hline
\end{tabular}

para asegurar la representatividad de los ítems seleccionados. Así se ha diseñado para cubrir un espectro de edades comprendidas entre los tres y los treinta y seis meses. Para ello se han construido un total de siete cuestionarios. Los cortes de edad han sido establecidos de forma convencional al estudiar los diferentes protocolos o programas de seguimiento de salud de los/as niños/as.

El número de ítems o hitos incorporados en cada cuestionario oscila entre los I I y los I6 ítems y oscila entre 5 minutos a los/as niños/as más pequeños y ro minutos en los preescolares. La selección de los ítems se ha realizado en base al principio de representatividad de la actividad dominante. Es decir, se han incluido los hitos evolutivos (ítems) que según los autores consultados el 90-95\% de los niños/as realizan al finalizar el periodo de tiempo analizado (Rico, 20I3; Alonso, 2014).

El SDPTD ${ }^{3}$ fue desarrollado con el principal objetivo de detectar cualquier problema que pudiera surgir en el desarrollo de un/a niño/a relacionado con un retraso en su desarrollo psicomotor con el objeto de comenzar las intervenciones necesarias lo antes posible

3. Sistema de Detección Precoz de Trastornos del Desarrollo.
(Alcantud, Alonso, \& Rico, 20I4). Se puede acceder a través del enlace http://acceso. uv.es/ProyectoC. Su desarrollo se realizó en U.I Acceso del Departamento de Psicología Evolutiva y de la Educación de la Universitat de València en colaboración con el CUDAP ${ }^{4}$ y la Conselleria de Sanitat de la Generalitat de Valenciana.

La mayoría de los ítems vienen acompañados de una breve explicación de la conducta que intenta evaluar el ítem. Complementariamente tiene un sistema de ayuda mediante el cual se presentan los ítems, con el complemento de un video donde un/a niño/a de edad normativa realiza la conducta objeto de la pregunta con el fin de facilitar la comprensión de la misma.

Al finalizar el cuestionario, automáticamente se genera un informe con los resultados obtenidos en formato PDF que se puede guardar o imprimir. Con este documento, una vez impreso, se puede acudir al pediatra para que verifique la información y, si se cree conveniente, se remita a los especialistas para una valoración más exhaustiva y si fuera necesario la remisión a un Centro de Atención Temprana para su evaluación y tratamiento.

4. Centre Universitari de Diagnòstic i Atencio Primerenca. 


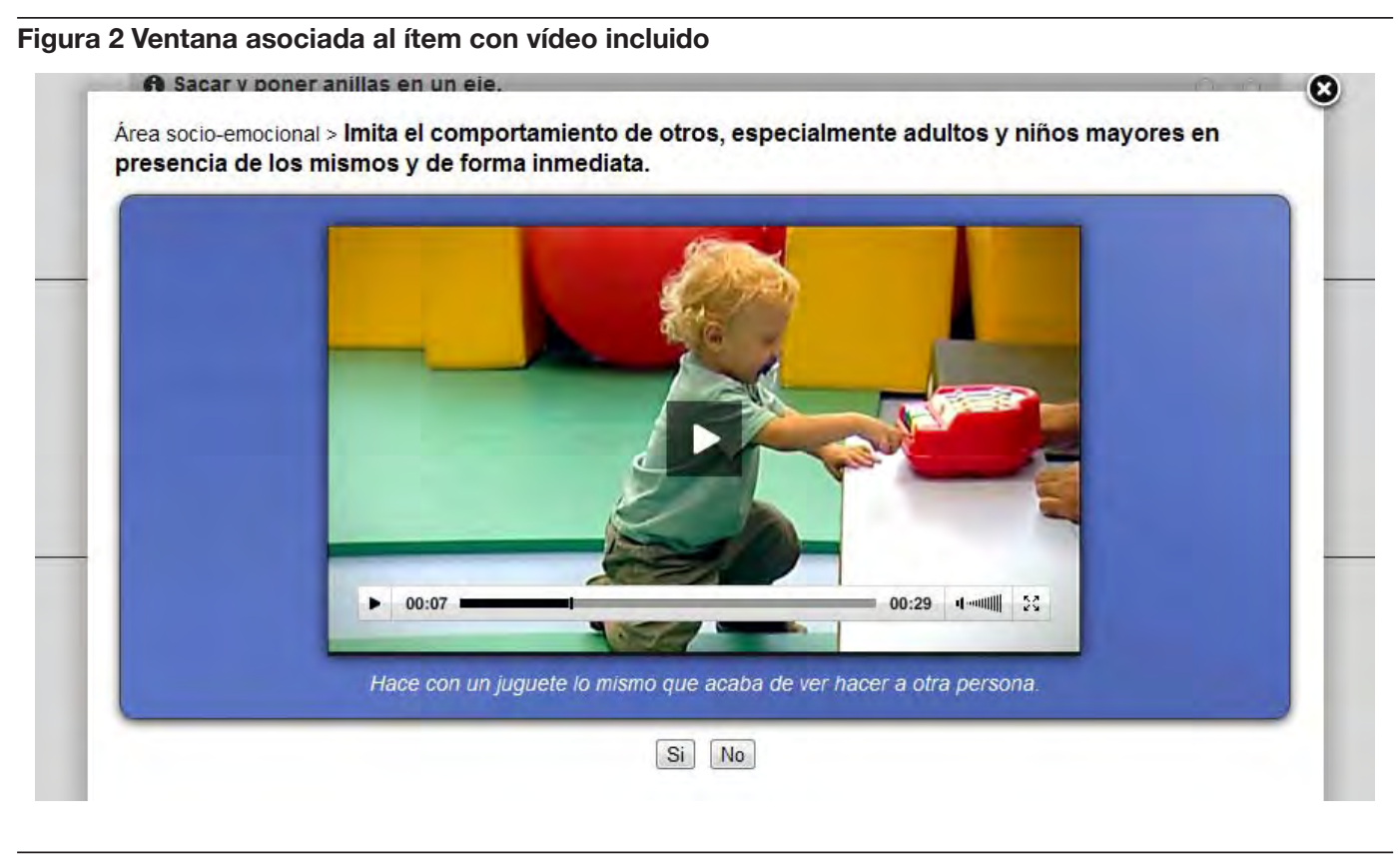

\subsection{Procedimiento}

Para el proceso de validación se contó con la colaboración de la Conselleria de Sanitat. El proyecto fue aprobado por el $\mathrm{CEIC}^{5} \mathrm{del} \mathrm{CSISP}^{6}$ el 27 de mayo del 20I I. El proceso de validación dada las grandes diferencias de edad se dividió en dos fases: en primer lugar, los cuestionarios de 18 meses hasta los 36 meses se administraron a una muestra de niños/as asistentes a centros educativos infantiles de la ciudad de Valencia (Rico, 20I3). En segundo lugar los cuestionarios de tres hasta los doce meses se aplicaron sobre una muestra de niños/as que acudieron en visita ordinaria a las consultas de Pediatría de Atención Primaria de los Centros de Salud de los departamentos del Hospital Clínico Universitario y Hospital Universitario de la Ribera en Alcira (Alonso, 2014).

En el primer caso se realizó una convocatoria a los centros infantiles de la ciudad de Valencia y acudieron a la misma un total de veinte centros educativos. Después de una acción formativa,

5. Comité Ético de Investigación Clínica.

6. Centro Superior de Investigación en Salud Pública. se instruyó a los profesionales para que distribuyeran los manuales de uso del sistema entre los padres/madres de los/as niños/as de la edad seleccionada invitándoles a participar. Una vez cumplimentados los cuestionarios, el documento demostrativo era entregado a los profesionales del centro que hicieron la verificación de la información (segundo nivel de cribado). Los/as niños/as que dieron positivo en las dos evaluaciones fueron derivados al CUDAP para realizar la evaluación por un especialista aplicándose como criterio "gold standard" la escala Merrill Palmer-R (Roid \& Sampers, 2004). Se constituyó un grupo control con niños/as de la misma edad y género a los que también se les aplicó la misma escala. Más detalles sobre el proceso de captación y muestreo se pueden encontrar en Rico (2013).

Para los cuestionarios de edades inferiores, dadas las dificultades de encontrar suficiente muestra en centros infantiles, se optó por acudir a los servicios de pediatría de los centros sanitarios de Atención Primaria. Los profesionales que colaboraron se encargaron de distribuir entre las familias atendidas los dípticos explicativos del sistema, solicitándoles 
que accedieran al mismo y contestaran al cuestionario de la edad de su hijo/a. Una vez realizada la primera evaluación por los/ as padres/madres, se les dio cita para que los propios pediatras se encargaran de validar las respuestas y, posteriormente, personal del equipo de investigación aplicó el test criterio o "gold standard". Igual que en la fase anterior, se eligió la escala de desarrollo Merrill-Palmer-R. El motivo de elegir este instrumento se debe a que la edad de aplicación se corresponde con la edad de aplicación del SDPTD, de forma que no es necesario utilizar más de un instrumento criterio en función de la edad, además, se trata de un instrumento de evaluación de espectro general que incluye todas las áreas del desarrollo.

\subsection{Participantes}

Aunque el objetivo de esta investigación no es un estudio poblacional ni un estudio epidemiológico, considerando que el proceso de captación no ha determinado la selección de los sujetos evaluados y por tanto, considerando la muestra como aleatoria, se ha estimado el error muestral a un nivel de confianza $\alpha=0,05 \%$. La aplicación de los cuestionarios de 3 a I 2 meses se realizó entre los meses de noviembre del 20 I I y julio del 20 I 2 y los cuestionarios de I 8 a 36 meses, se administraron desde marzo a Julio del 20I I.

Un año después de la aplicación, se realizó un seguimiento telefónico a las familias para conocer el estado de desarrollo de los/as niños/ as. Esta información, junto con la recogida en la anamnesis de cada caso, será utilizada para determinar la validez diagnóstica del SDPTD (Rico, 20I3; Alonso, 20I4).

\section{Resultados}

Los cuestionarios han sido analizados siguiendo un sistema de análisis psicométrico convencional (Tornimberi \& Perez, 2008; Muñiz, 2003; Muñiz, Fidalgo, Garcia-Cueto, Martinez \& Moreno, 2005). El criterio de calidad para estos cuestionarios se basa en el valor de diferentes indicadores psicométricos como la fiabilidad, validez, sensibilidad y especificidad entre otros (Camp, 2006; Johnson \& Marlow, 2006). Inicialmente y con la finalidad de determinar si los ítems cumplen el criterio de selección, es decir que representen conductas usuales en niños/as de la edad superior al 90\%, se calcularon los índices de dificultad $(\mathrm{P})$.

Como se puede observar en la tabla 3 , la mayoría de cuestionarios cumplen el criterio de que cada ítem representa un hito evolutivo que cumple como mínimo el 90-95\% de la población. El cuestionario que mejor cumple este criterio es el de tres y veinticuatro meses y el que peor lo cumple el de 36 meses.

\section{Tabla 2. Distribución de la muestra en función de la edad}

\begin{tabular}{|c|c|c|c|c|c|c|c|c|}
\hline & & \multicolumn{7}{|c|}{ Edad en meses } \\
\hline & & 3 & 6 & 9 & 12 & 18 & 24 & 36 \\
\hline \multirow{2}{*}{ 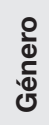 } & Varones & 47 & 47 & 59 & 57 & 44 & 84 & 34 \\
\hline & Mujeres & 56 & 45 & 38 & 37 & 49 & 86 & 45 \\
\hline & Total & 103 & 92 & 97 & 94 & 93 & 170 & 79 \\
\hline \multicolumn{2}{|c|}{ Error Muestral } & 0.0675 & 0.0715 & 0.0696 & 0.0707 & 0.0707 & 0.052 & 0.077 \\
\hline
\end{tabular}


Tabla 3. Porcentaje de ítems constantes y por debajo el criterio $\mathrm{p}=0,90$

\begin{tabular}{|l|c|c|c|c|c|c|c|c|}
\hline & \multicolumn{2}{|c|}{3 meses } & \multicolumn{2}{c|}{6 meses } & \multicolumn{2}{c|}{9 meses } & \multicolumn{2}{c|}{12 meses } \\
\hline \% Ítems & Padres & Prof & Padres & Prof & Padres & Prof & Padres & Prof \\
\hline $\mathrm{P}<0.90$ & 0 & 0 & 18,18 & 0 & 28,57 & 14,29 & 25,00 & 12,50 \\
\hline Constante & 33,33 & 25,00 & 18,18 & 54,55 & 28,57 & 21,43 & 6.25 & 18,75 \\
\hline
\end{tabular}

\begin{tabular}{|l|c|c|c|c|c|c|}
\hline & \multicolumn{2}{|c|}{18 meses } & \multicolumn{2}{c|}{ 24 meses } & \multicolumn{2}{c|}{36 meses } \\
\hline \% Ítem & Padres & Prof & Padres & Prof & Padres & Prof \\
\hline $\mathrm{P}<0.90$ & 0 & 6.66 & 0 & 0 & 0 & 0 \\
\hline Constante & 13.33 & 20.00 & 28.57 & 14.28 & 26.09 & 56.52 \\
\hline
\end{tabular}

\subsection{Fiabilidad}

La consistencia interna de los test se mide utilizando el coeficiente Alfa de Cronbach (Cronbach, I95I; Tornimberi \& Perez, 2008; Muñiz, 2003; Viladrich, Doval \& Prat, 2005).

En la tabla 4 se presentan una síntesis de los índices de fiabilidad calculados para los siete cuestionarios. Obsérvese como en general el cuestionario con las respuestas de los/as padres/madres corregidas por los profesionales tienen más fiabilidad que la de los/as padres/ madres, con excepción del cuestionario de 6 meses. Se observa que la fiabilidad es media y aceptable, con excepción del cuestionario de 3 meses (.44) y el de 6 meses (contestado por los profesionales). No obstante, debemos tener en cuenta que el Alfa de Cronbach mide consistencia interna y su valor se relaciona con el número de ítems (longitud de la prueba) y la dispersión de las puntuaciones. Como los ítems de los cuestionarios fueron seleccionados buscando que el 90-95\% de la población lo resolviera, la consecuencia es que existe poca dispersión en la respuesta lo que produce índices de fiabilidad más bajos. No obstante, está pendiente en una segunda aplicación (versión 2.o) la introducción de un mayor número de ítems en estos cuestionarios y el cálculo de fiabilidad por test-retest en los que esperamos que los resultados sean algo más elevados siguiendo la tendencia de las correlaciones entre los resultados de los padres y los profesionales.

El uso de herramientas de cribado por los/ as padres/madres, aunque claramente reduce coste, genera una preocupación general sobre la fiabilidad de las respuestas de los/as padres/ madres respecto al desarrollo de sus hijos/

Tabla 4. Valores de fiabilidad calculados por el Alpha de Cronbach para cada uno de los cuestionarios

\begin{tabular}{|l|c|c|c|c|c|c|c|}
\hline & 3 meses & 6 meses & 9 meses & 12 meses & 18 meses & 24 meses & 36 meses \\
\hline Padres & .44 & .66 & .54 & .68 & .82 & .797 & .584 \\
\hline Profesionales & .77 & .44 & .64 & .68 & .831 & .85 & .60 \\
\hline
\end{tabular}

Tabla 5. Correlaciones entre las respuestas de los padres y las puntuaciones de los profesionales

\begin{tabular}{|l|l|l|l|l|l|l|l|}
\hline$r_{x y}$ & 3 meses & 6 meses & 9 meses & 12 meses & 18 meses & 24 meses & 36 meses \\
\hline padres/prof & 0.846 & 0,903 & 0.872 & 0.900 & 0.98 & 0.93 & 0.459 \\
\hline
\end{tabular}


as (Sheehan, I988). Esta preocupación dio pie a muchas investigaciones alrededor de su validez. En la mayoría de las investigaciones se informa de un acuerdo consistente entre las evaluaciones de profesionales y de padres/ madres, en particular cuando estos últimos respondían a cuestiones sobre el desarrollo actual (Squires, Potter, \& Bricker, I 998). En este caso, las puntuaciones obtenidas por los profesionales, son el resultado de la evaluación de los resultados de los/as padres/madres, por tanto, no son dos evaluaciones totalmente independientes. No obstante, las correlaciones tal como se muestra en la tabla 5 son muy altas. Se puede concluir este punto diciendo que las observaciones de los profesionales pueden cambiar en magnitud pero no en dirección.

\subsection{Validez concurrente}

La validez hace referencia a la capacidad de un instrumento de medición para cuantificar de forma significativa y adecuada el rasgo para cuya medición ha sido diseñado (Viladrich, Doval, \& Prat, 2005; Garcia, I993; Tornimberi \& Perez, 2008). La constatación de la validez de un instrumento de detección no debería ofrecer mayores problemas si el criterio de contraste está bien definido. En general, dados los problemas de definición, se suele optar por utilizar diferentes criterios. En primer lugar, se presenta la validez concurrente (correlación entre el nuevo test y un test previamente validado), en segundo lugar se presenta la validez predictiva y por último la validez diagnóstica.

En el caso de la validez concurrente, el criterio utilizado en este estudio ha sido la escala de desarrollo MP-R (Roid \& Sampers, 2004). Si se observa la tabla 6 , salvo el caso del cuestionario de doce meses, las correlaciones entre las respuestas de los/as padres/madres y profesionales y los resultados de la MP-R son medios y mediosaltos y todos estadísticamente significativos.

\subsection{Validez predictiva/discriminante}

Para el estudio de la validez predictiva o discriminante se crearon dos grupos en cada nivel de edad, por una parte los/as niños/as que obtuvieron una puntuación superior al punto de corte de la MP-R en el Índice General, y por otro lado, los que obtuvieron una puntuación inferior a este punto de corte. (Criterio propio de la MP-R de tener una puntuación en el $\mathrm{IG}<85$ ).

Obsérvese que la bondad de clasificación sobre los resultados de la MP-R en base a los ítems del SDPTD es muy elevado en todos los casos. Este dato junto con el apartado anterior hace concluir que la aplicación del SDPTD equivaldría a la aplicación de la MP-R.

Tabla 6. Índices de correlación entre las puntuaciones dadas por los padres y profesionales y el Índice General Cognitivo obtenido de la MP-R

\begin{tabular}{|l|c|c|c|c|c|c|c|}
\hline & 3 meses & $\mathbf{6}$ meses & 9 meses & 12 meses & 18 meses & 24 meses & 36 meses \\
\hline$r_{x y}$ padres - MP-R & $0,521^{\star \star}$ & $0,300^{\star \star}$ & $0,735^{\star \star}$ & 0,168 & $0.804^{\star \star}$ & $0.727^{\star \star}$ & $0.461^{\star *}$ \\
\hline$r_{x y}$ prof - MP-R & $0,622^{\star \star}$ & $0,415^{\star \star}$ & $0,750^{\star \star}$ & 0,132 & $0.795^{\star \star}$ & $0.720^{\star \star}$ & 0.353 \\
\hline
\end{tabular}

Tabla 7. \% de clasificación correcta sobre los grupos definidos por el criterio de la MP-R en base a las funciones discriminantes calculadas a partir de las respuestas al SDPTD de los profesionales

\begin{tabular}{|l|c|c|c|c|c|c|c|}
\hline & $\mathbf{3}$ meses & $\mathbf{6}$ meses & $\mathbf{9}$ meses & $\mathbf{1 2}$ meses & $\mathbf{1 8}$ meses & $\mathbf{2 4}$ meses & $\mathbf{3 6}$ meses \\
\hline $\begin{array}{l}\text { \% clasificación } \\
\text { correcta con funciones } \\
\text { discriminantes }\end{array}$ & 99,00 & 98,9 & 96,9 & 96,8 & 100 & 96,1 & 96.0 \\
\hline
\end{tabular}




\subsection{Validez diagnóstica}

El concepto de validez predictiva o de pronóstico también hace referencia cuando el criterio no es una medida, que es un estado que queremos predecir con este test. En este caso sería el diagnóstico médico de padecer o pertenecer a alguna población de riesgo, en consecuencia se le suele denominar "Validez Diagnóstica” como la capacidad de predicción que tiene un test para predecir un diagnóstico como estado discreto.

En España, según la Encuesta sobre Discapacidades, Deficiencias y Estado de Salud realizada por el Instituto Nacional de Estadística, nos sitúan ante más de un $7 \%$ de niños/as que precisan seguimiento y/o tratamiento en los centros de atención temprana. En la aplicación piloto no sería esperable un porcentaje de detección superior al Io\% de la muestra. En un estudio sobre una población infantil de 472 niños/as nacidos entre I 996 y 2002 y seguidos en la consulta de Atención Primaria (Centro de Salud de Mendillorri) en la ciudad de Pamplona (Navarra), se encontró que el 8,68\% de niños/as tenían algún trastorno o riesgo de padecerlo (Galbe Sanchez-Ventura, 20I3). En el caso objeto de investigación se ha obtenido información de tres vías: por parte del cuestionario (contestado por padres/madres y profesionales), por el diagnóstico conocido recogido en la anamnesis y por último, el estado del niño/a un año después de la aplicación.
Los resultados obtenidos son diferentes en función de cada corte de edad, como se puede observar en la tabla 8. Para el corte de tres meses el porcentaje de población detectado por los profesionales (cuestionario de padres/ madres corregido por los profesionales) alcanza un $7,77 \%$ frente al $5,83 \%$ de casos conocidos y el $6,8 \%$ de casos conocidos un año después. Existe posiblemente una sobreestimación de casos detectados tanto por los/as padres/madres como por los profesionales. Se debe hacer una reflexión sobre esta cuestión. Es posible que, por el procedimiento de captación seguido, se haya producido un efecto llamada, de forma que acudieran más las familias que tenían niños/as con problemas y menos las de desarrollo típico (Alonso, 20I4).

En la misma línea, si observamos los valores de sensibilidad y especificidad de la clasificación con el criterio de diagnóstico (conocido un año después), se observa un comportamiento muy aceptable de las puntuaciones de los profesionales (acumula la de los padres/madres) y en algún caso, mejorado por las funciones discriminantes. Es importante recordar las diferencias procedimentales existentes en el proceso muestral entre los cuatro primeros segmentos de edad y los tres últimos. En estos tres últimos solo se aplicó la escala criterio a los/as niños/as con resultados positivos y a un grupo control, consecuentemente los índices de sensibilidad y especificidad se ven afectados y no tienen valor poblacional.

Tabla 8 Valores comparativos de resultados según segmentos de edad

\begin{tabular}{|l|c|c|c|c|}
\hline \multirow{2}{*}{} & \multicolumn{4}{|c|}{ \% casos positivos } \\
\cline { 2 - 5 } & Padres & Profesionales & Diagnóstico Previo & 1 año después \\
\hline 3 meses & 15,53 & 7,77 & 5,83 & 6,80 \\
\hline 6 meses & 27,17 & 14,13 & 4,35 & 4,35 \\
\hline 9 meses & 41,23 & 23,71 & 13,40 & 19,59 \\
\hline 12 meses & 63,83 & 46,81 & 15,96 & 15,96 \\
\hline 18 meses & 15,53 & 7,77 & 5,83 & 6,80 \\
\hline 24 meses & 27,17 & 14,13 & 4,35 & 4,35 \\
\hline 36 meses & 63,83 & 46,81 & 15,96 & 15,96 \\
\hline
\end{tabular}


Tabla 9 Tabla síntesis con los valores de sensibilidad y especificidad para el criterio de un año después

\begin{tabular}{|c|c|c|c|c|c|c|c|}
\hline & 3 meses & 6 meses & 9 meses & 12 meses & 18 meses & 24 meses & 36 meses \\
\hline \multicolumn{8}{|c|}{ Resultados para puntuación de padres } \\
\hline Sensibilidad & 0.714 & 0.75 & 1.00 & 1.00 & 1.00 & 0.875 & 0.80 \\
\hline Especificidad & 0.873 & 0.87 & 0.81 & 0.36 & 0.36 & 0.44 & 0.687 \\
\hline Valor Predictivo Positivo & 0.357 & 0.25 & 0.65 & 0.29 & 0.29 & 0.33 & 0.444 \\
\hline Valor Predictivo Negativo & 0.968 & 0.98 & 1.00 & 1.00 & 1.00 & 0.916 & 0.916 \\
\hline \multicolumn{8}{|c|}{ Resultados para puntuación de profesionales } \\
\hline Sensibilidad & 0.571 & 0.50 & 0.90 & 1.00 & 1.00 & 0.875 & 0.80 \\
\hline Especificidad & 0.971 & 0.94 & 0.98 & 0.59 & 0.59 & 0.44 & 0.562 \\
\hline Valor Predictivo Positivo & 0.666 & 0.33 & 0.94 & 0.39 & 0.39 & 0.33 & 0.363 \\
\hline Valor Predictivo Negativo & 0.958 & 0.97 & 0.96 & 1.00 & 1.00 & 0.916 & 0.90 \\
\hline \multicolumn{8}{|c|}{ Resultados función discriminante } \\
\hline Sensibilidad & 0.571 & 0.75 & 0.70 & 0.66 & 0.86 & 0.875 & 0.80 \\
\hline Especificidad & 1.00 & 0.90 & 0.98 & 0.94 & 0.77 & 0.44 & 0.687 \\
\hline Valor Predictivo Positivo & 1.00 & 0.30 & 0.93 & 0.77 & 0.50 & 0.33 & 0.444 \\
\hline Valor Predictivo Negativo & 0.959 & 0.98 & 0.90 & 0.91 & 0.95 & 0.916 & 0.916 \\
\hline
\end{tabular}

En otras publicaciones de mayor extensión (Alcantud, Alonso, \& Rico, 20I4), se presentan resultados de las Curvas ROC para la determinación de diferentes puntos de corte. De estos resultados se desprende que el sistema en su conjunto muestra un comportamiento adecuado, aunque como resulta obvio, mejorable. Para evitar el coste emocional que puede significar para una familia, la noticia de que su hijo/a dio positivo en un sistema de detección de trastornos del desarrollo y con la finalidad de garantizar el mínimo de falsos positivos, se propone aplicar puntos de corte múltiples. Se trata de calcular dos puntos de corte que determinen una zona de seguridad donde el impacto de los falsos positivos se aminore. En la tabla ıo se resume los resultados obtenidos para los siete cuestionarios. Obsérvese como los casos positivos sobre el criterio diagnóstico un año después, se distribuyen en la mayoría de los casos entre la categoría de Riesgo alto y Riesgo moderado. El número de falsos negativos es muy bajo, incluso en el cuestionario de doce meses es cero.

Tabla 10 Distribución del número de casos según riesgo calculado por las respuestas de los profesionales y el diagnóstico un año después

\begin{tabular}{|l|c|c|c|c|c|c|c|c|c|c|c|c|c|c|}
\hline & \multicolumn{2}{|c|}{3 meses } & $\mathbf{6}$ meses & \multicolumn{2}{|c|}{$\mathbf{9}$ meses } & \multicolumn{2}{|c|}{$\mathbf{1 2}$ meses } & $\mathbf{1 8}$ meses & \multicolumn{2}{|c|}{$\mathbf{2 4}$ meses } & \multicolumn{2}{c|}{$\mathbf{3 6}$ meses } \\
\hline RIESGO & SI & NO & SI & NO & SI & NO & SI & NO & SI & NO & SI & NO & SI & NO \\
\hline Alto & 4 & 0 & 2 & 6 & 13 & 0 & 7 & 2 & 5 & 2 & 2 & 4 & 2 & 3 \\
\hline Moderado & 0 & 2 & 1 & 4 & 5 & 1 & 8 & 24 & 2 & 3 & 5 & 10 & 2 & 2 \\
\hline Sin Riesgo & 3 & 69 & 2 & 78 & 2 & 57 & 0 & 30 & 0 & 1 & 1 & 11 & 1 & 10 \\
\hline
\end{tabular}




\section{Conclusión}

En definitiva, se puede concluir que el SDPTD es un sistema de detección para detectar los trastornos del desarrollo que muestra una adecuada fiabilidad y su validez. La validez ha quedado demostrada tanto al compararla de forma concurrente con la escala MP-R, como a nivel diagnóstico al comparar sus resultados con los diagnósticos conocidos y el seguimiento un año después. Esta es una primera versión de los cuestionarios que será mejorada en las siguientes revisiones. A nivel de la usabilidad, el sistema en general ha sido valorado como muy sencillo y accesible.

Como líneas de trabajo futuro, como ya se ha dicho en el texto, se deben mejorar los instrumentos, mejorando su redacción y los videos de apoyo a cada uno de los ítems. Se debe valorar también que además de ser un instrumento diagnóstico, también tiene un valor educativo, dando información a los padres sobre cuáles son los hitos evolutivos de sus hijos/as.

En cuanto a la forma de respuesta, es posible que la respuesta dicotómica provoque una respuesta forzada que en ocasiones no es del todo correcta. Se optó por esta solución por ser fácil de valorar, no obstante, muchos padres/ madres comunican que sus hijos/as lo hacían en ocasiones, pero no siempre, o simplemente decían que no lo habían observado. Se deben incorporar estas opciones de respuesta. Cuando se trata de responder si un/a niño/a "señala los objetos cuando se le nombran", la respuesta no solo será sí o no, bien podría ser "a veces" o "casi siempre". Este tipo de respuestas abren la posibilidad a utilizar la Lógica Difusa para un mejor procesamiento de la herramienta de evaluación. Se han desarrollado algunas herramientas para diagnosticar trastornos del desarrollo a edad temprana, tal es el caso del sistema KBS (Knowledge Based Screener) basado en reglas si-entonces. Por ejemplo, se ha desarrollado un sistema experto experimental que ayudan a identificar el/la niño/a con problemas (Veeraraghavan \& Srinivasan, 2007). En el caso de los Trastornos del Espectro Autista, existen estudios en los que se aplican estos principios basándose en una lista de supuestos y el MCHAT (Kannappan, Tamilarasi \& Papageorgiou, 20I I). Está en una línea de trabajo que linda entre el desarrollo metodológico, el desarrollo tecnológico y la aplicación diagnóstica.

Por último, la versión actual del SDPTD está abierta y lo único que se requiere es introducir la fecha de nacimiento y el distrito postal de residencia. Se debe cerrar el acceso y requerir un "login" controlado por nombre de usuario y contraseña. Esta opción se descartó en su momento puesto que obligaba, según la LOPD (Ley I5/I999 de I3 de diciembre), a publicar la base de datos y mantener unos sistemas de seguridad que para el proceso de investigación resultaban caros e innecesarios. Ahora, por el contrario, puesto a hacer público el uso de este instrumento, se cree necesario guardar los resultados de cada cuestionario e incluso arbitrar un sistema que, automáticamente, cuando la puntuación del/la niño/a esté en una zona de riesgo, avise a la familia cuando llegue la edad para cumplimentar el siguiente cuestionario o solicitar información de confirmación de diagnóstico. Una herramienta de estas características nos permitiría, con un coste razonable, poder plantearnos estudios incluso de ámbito nacional e internacional. 


\section{Referencias bibliográficas}

Aguirre, J. (200I). La actividad fisica del niño: de cero a tres años. Pamplona: Instituto Navarro de Deporte y Juventud.

Alcantud, F. et al. (2014). Sistema de Detección Precoz de Trastornos del Desarrollo (SDTP): Construcción, validación y manual de uso (versión I.O). Madrid: En prensa.

Alonso, Y. (20I4). Validación del Sistema de Detección Precoz de Trastornos del Desarrollo (SDPTD). 3 a I2 meses. Valencia: Universitat de València.

Alvarez, M. y Giner, A. (2007). “Desarrollo Psicomotor". Revista de Pediatria y Atención Primaria, 59-66.

Alvarez, M. et al. (2009). "Importancia de la vigilancia del desarrollo psicomotor por el pediatra de Atención Primaria: Revisión del tema y experiencia de seguimiento en una consulta de Navarra”. Revista Pediatrica de Atención Primaria, II (4I): 65-87.

Artigas-Pallares, J. (20II). “Trastornos del neurodesarrollo: Conceptos básicos”. En J. Artigas-Pallares, y J. Narbona: Trastornos del Neurodesarrollo, 3-I6. Barcelona: Viguera Ediciones.

Aylward, G. (I995). Bayley Infant Neurodevelopmental Screener. San Antonio: Psychological Corporation.

Barnett, S. (I995). "Long term effects of early childhood programs on cognitive and school outcomes". Future Child, 25-50.

Canal, R. et al. (2006) "La deteccion precoz del autismo". Intervención Psicosocial, I5 (I): 29-47. http://scielo.isciii.es/pdf/inter/vi 5 nI/ vi 5niao3.pdf>.

Camp, B. W. (2006). "What the clinician really needs to know: Questioning the clinicl usefulness of sesitivy and specificity in studies of screening test". Journal of Developmental and Behavioral Pediatrics, 27: 226-230.

Casto, G. y Mastropieri, M. (I986). “The Efficacy of Early Intervention Programs: A Meta-
Analysis". Exceptional Children, 52 (5):

Cronbach, L. (I95 I). "Coefficient alpha and the internal structure of tests". Psychometrika, 297-334.

Fernández, E. (1988). El desarrollo psicomotor de I.702 niños de o a 24 meses de edad. [Tesis doctoral]. Universidad de Barcelona.

Fernández, E. et al. ( I991). Tabla de desarrollo Haizea-Llevant. Servicio Central de Publicaciones del Gobierno Vasco: Vitoria.

Frankenburg, W. et al. (I992). "The Denver II: A major revision and restandardization of the Denver Developmental Screenig Test". Pediatrics, 89: 91-97.

Galbe, J. (2013). Atención orientada al desarrollo y supervisión del desarrollo psicomotor (en línea). 〈http://www.aepap.org/previnfad/ psicomotor.htm». Grupo Previnfand.

Garcia, E. (I993). Introducción a la Psicometría. Madrid: Alianza.

García, J. N. (I999): “Intervención Psicopedagógica en los Trastornos del Desarrollo. Conceptualización, ámbito y modelo Integral”. En García Sánchez, J. N. (Ed.): Intervención Psicopedagógica en los Trastornos del Desarrollo. Ed. Pirámide. Madrid.

Gesell, A. (I974). Psicología Evolutiva de I a I6 años. Buenos Aires: Paidos.

Glascoe, F. y Macías, M. (2003). "How you can implement the AAP's new policy on developmental and behavioural screening". Contemporary Pediatrics, 85-102.

Guralnick, M. (1989,I997). Recents developments in Early Intervention Efficacy Research:Implicantions for family involvement.

Guralnick, M. (I998). "The effectiveness of early intervention for vulnerable children: A developmental perspective". American Journal on Mental Retardation, IO2: 319-345. 
Guralnick, M. J. y Bennett, F. C. (1987). The effectiveness of early intervention for atrisk and handicapped children. New York: Academic Express.

Hamilton, S. (2006). Screening for developmental delay: Reliable, easy-to-use tools. Journal of Familiy Practice, 5 5: 4I 5-422.

Hickson, G. et al. (1983). "Concerns of mothers seeking care in private pediatric offices: Opportunities for expanding services". Pediatrics, 72: 619-624.

Hix-Small, H. et al. (2006). "Impact of implementing Developmental Screeningo at I 2 and 24 months in a Pediatric Practice". Pediatrics, $38 \mathrm{I}-388$.

Horowitz, F. (1989). "Using developmental theory to guide the search for the effects of biological risk factors on the developmental of children". American Journal of Clinical Nutrition, 50 : 589-95.

Illingworth, R. (I992). El desarrollo del lactante $y$ del niño. Churchill Livingtone. Edición Española Alhambra Longman.

Johnson, S. y Marlow, N. (2006). Developmental screen or developmental testing? Early Human Develpment, 82,I73-183.

Kannappan, A. et al. (20I I). "Analyzing the performance of fuzzy cognitive maps with nonlinear hebbian learning algoritm in predicting autistic disorder". Journal Expert Systems with Applications, 38 (3): I 282-I 292.

McCormick, M. et al. (2006). "Early intervention in Low Birth Weight Premature Infants: Results at I 8 years of age for the Infant Health and Development Program”. Pediatrics, I I 7 : 77I-780.

Melchers, P. et al. (2003). Erweterte Vorsorgeuntersuchung. Leiden : PITS.

Muñiz, J. (2003). Teoria Clasica de los tests. Madrid: Pirámide.

Muñiz, J. et al. (2005). Análisis de items. Madrid: La Muralla.

Olhweiler, L. et al. (2002). "Estudio del desarrollo psicomotor en pacientes prematuros durante el primer año de vida". Revista de Neurologia, $35(8), 727-730$.

Osofsky, J. (1987). Handbook of infant development. New York: Willey.

Palfrey, J. et al. (I987). "Early identification of children's special need: a study in five metropolitan communities". Journal of Pediatrics, 65 I-659.

Reynolds, A. et al. (200I). "Long term effects of an early childhood intervention on educational achievement and juvenile arrest". Journal of the American Medical Association, 2339-2346.

Rico, D. (2013). Construcción y Validación de un Sistema de Detección Precoz de los Trastornos del Desarrollo (SDPTD): I 8 a 36 meses. Valencia: Universitat de València.

Roid, G., y Sampers, J. (2004). Scales of MerrillPalmer-Revised. Wooddale,IL: Stoelting Co.

Rydz, D., y Col, E. (2006). “Screenig for developmental delay in teh settingo of a community pediatric clinic: a prospective assessment of parent-report questionnaires". Pediatrics, II78-II85.

Schopler, E. (1983). "New developments in the definition and diagnosis of autism". En Lahey, B.B. y Kazdin, A.E. (Eds): Advances in clinical child psychology, 6: 93-I 27. New York, Plenum Publishing Corporation

Sheehan, R. (I988). "Involvement of parents in early childhood assessment". En T. Wachs y R. Sheehan (Eds): Assessment of young developmentally disabled children, 75-90.

Shonkoff, J. P. y Hauser-Cram, P. (I987). "Early Intervention for Disabled Infants and Their Families: A Quantitative Analysis". Pediatrics, $80(5): 650-58$.

Sices, L. et al. (2003). "How do primay care physicians identify young children with developmental delays? A National Survey". Developmental Behavioral Pediatircs, 409-4I7.

Smith, R. (I978). “The use of developmental screening tests by primary care pediatricians". Journal of Pediatrics, 524-527.

Squires, J. et al. (I998). "Parent-completed Developmental questionnarires: Effectiveness 
with low and middke income parents". Early Childhood Research Quaterly, 345-353.

Tornimberi, S., y Perez, E. (2008). Introducción a la Psicometría. Buenos Aires: Paidos.
Veeraraghavan, S. y Srinivasan, K. (2007).

"Exploration of autism using expert systems". Information Technology ITNG'07, 26I-264.

Viladrich, C. et al. (2005). Psicometria. Barcelona: Universitat Oberta de Catalunya. 